\title{
The influence of enzyme-resistant starch on cholesterol metabolism in rats fed on a conventional diet
}

\author{
K. Vanhoof and R. De Schrijver* \\ Laboratory of Nutrition, Catholic University of Leuven, Kard. Mercierlaan 92, 3001 Leuven, Belgium \\ (Received 6 August 1997 - Revised 10 February 1998 - Accepted 25 February 1998)
}

\begin{abstract}
Male Wistar rats were fed on a conventional diet containing normal corn starch or $6 \%$ enzymeresistant starch originating from either raw or retrograded high-amylose corn starch. Furthermore, the diets were either cholesterol-free or contained $1 \%$ cholesterol and $0.1 \%$ cholic acid. The main objective of this study was to investigate whether the addition of enzyme-resistant starch to a rat conventional diet had any effect on cholesterol metabolism. Therefore, plasma and liver cholesterol concentrations, plasma HDL:LDL cholesterol ratios and neutral steroid and bile acid excretion were determined. No significant effect of enzyme-resistant starch feeding on plasma and liver cholesterol concentrations was found. However, consumption of raw or retrograded high-amylose corn starch resulted in a decrease in esterified and total liver cholesterol concentrations of 24 and $22 \%$, respectively. This was accompanied by a reduction in plasma esterified and total cholesterol levels of $4 \%$ and a tendency to higher daily faecal coprostanol and total bile acid excretion.
\end{abstract}

\section{Resistant starch: Cholesterol metabolism: Rats}

A number of recent studies (de Deckere et al. 1993, 1995; Younes et al. 1995; Vanhoof \& De Schrijver, 1997) show that enzyme-resistant starch (RS) might have a hypocholesterolaemic effect, both in normo- and hypercholesterolaemic rats. This effect may be caused by lower cholesterol absorption, increased neutral steroid and bile acid excretion or increased synthesis of fermentation products like propionic acid, which in turn may decrease cholesterol synthesis in the liver (Chen et al. 1984). Yet, this last factor is considered controversial as in vivo propionate concentrations might not be high enough to decrease the activity of hydroxy-methylglutaryl-CoA reductase (EC $1 \cdot 1.1 \cdot 88$ ), the rate-limiting enzyme in hepatic cholesterol synthesis (Illman et al. 1988; Beaulieu \& McBurney, 1992).

In this study the effect of RS on cholesterol metabolism in normo- as well as hypercholesterolaemic rats was investigated by measuring plasma cholesterol concentrations and faecal steroid excretion. High-amylose corn starch and retrograded high-amylose corn starch were used as sources of RS types 2 and 3 respectively. In contrast with the above mentioned studies which were performed with semi-synthetic diets, we wanted to test the effect of both RS sources when added to a conventional diet. Consequently, we could examine whether the hypocholesterolaemic effect of RS that was observed in our previous study with semi-synthetic diets (Vanhoof \& De Schrijver, 1997) was influenced by diet composition.

\section{Materials and methods}

Enzyme-resistant starch sources

High-amylose corn starch $\left(\right.$ Hylon VII $^{\circledR}$ ) and retrograded high-amylose corn starch (Novelose ${ }^{\circledR}$ ) were used as sources of RS types 2 and 3 respectively. Fibre sources were obtained from Unilever (Vlaardingen, The Netherlands) and National Starch and Chemical Company (Neustadt, Germany). Hylon VII ${ }^{\circledR}$ contained $55.4 \%$ and Novelose ${ }^{\circledR}$ $41.1 \%$ RS. Normal corn starch (Meritena A, Amylum, Belgium) was used as a reference.

\section{Animals and diets}

All diets contained the same amounts of barley, wheat, cassava and soyabean meal as the major ingredients (Table 1). Two normocholesterolaemic test diets were devised to contain $6 \%$ RS type 2 or 3 . Therefore, $10.8 \%$ Hylon VII ${ }^{\circledR}$ (RS2-) or $14.6 \%$ Novelose $^{\circledR}$ (RS3-) were added at the expense of the normal corn starch in the normocholesterolaemic control diet $(\mathrm{C}-)$. Three other diets (C+, RS2+ and RS3+) were devised which were similar to the preceding ones, except that they contained $1 \%$ cholesterol and $0.1 \%$ cholic acid replacing diatomaceous earth. Cholic acid was added to enhance the hypercholesterolaemic effect of the cholesterol feeding.

The animal care procedures were conducted under 
Table 1. Composition of the diets $(\mathrm{g} / \mathrm{kg})$

\begin{tabular}{|c|c|c|c|c|c|c|}
\hline Ingredient & $\mathrm{C}-$ & RS2- & RS3- & $\mathrm{C}_{+}$ & $\mathrm{RS} 2+$ & RS3+ \\
\hline Barley & 371 & 371 & 371 & 371 & 371 & 371 \\
\hline Wheat & 173 & 173 & 173 & 173 & 173 & 173 \\
\hline Cassava & 112 & 112 & 112 & 112 & 112 & 112 \\
\hline Soyabean meal & 43 & 43 & 43 & 43 & 43 & 43 \\
\hline Corn starch & 203 & 95 & 57 & 203 & 95 & 57 \\
\hline Hylon VII ${ }^{\Theta_{*}}$ & - & 108 & - & - & 108 & - \\
\hline Novelose ${ }^{\circledR} \dagger$ & - & - & 146 & - & - & 146 \\
\hline Cellulose & 16 & 16 & 16 & 16 & 16 & 16 \\
\hline Sucrose & 13 & 13 & 13 & 13 & 13 & 13 \\
\hline Corn oil & $12 \cdot 5$ & $12 \cdot 5$ & $12 \cdot 5$ & $12 \cdot 5$ & $12 \cdot 5$ & $12 \cdot 5$ \\
\hline \multicolumn{7}{|l|}{ Vitamin, mineral and } \\
\hline Diatomaceous earth & 11 & 11 & 11 & - & - & - \\
\hline Cholesterol & - & - & - & 10 & 10 & 10 \\
\hline Cholic acid & - & - & - & 1 & 1 & 1 \\
\hline Total dietary fibreł & 177 & 191 & 219 & 190 & 196 & 224 \\
\hline
\end{tabular}

C, control diet without added resistant starch (RS); RS2, diet with $6 \%$ RS type 2; RS3, diet with $6 \%$ RS type 3;--, diet without added cholesterol and cholic acid; +, diet with $1 \%$ cholesterol and $0.1 \%$ cholic acid.

* Hylon VII ${ }^{\circledR}$, high-amylose corn starch obtained from Unilever, Vlaardingen, The Netherlands and National Starch and Chemical Company, Neustadt, Germany.

† Novelose ${ }^{\circledR}$, retrograded high-amylose corn starch obtained from Unilever, Vlaardingen, The Netherlands and National Starch and Chemical Company, Neustadt, Germany.

‡ Measured according to the procedure of Prosky \& Lee (1992).

Table 2. Plasma cholesterol concentrations* (Mean values with their standard errors for eight animals)

\begin{tabular}{|c|c|c|c|c|}
\hline \multirow[b]{2}{*}{ Diet } & \multicolumn{3}{|c|}{ Cholesterol concentration (mmol/l) } & \multirow{2}{*}{$\begin{array}{l}\text { HDL:LDL } \\
\text { cholesterol } \\
\text { ratio }\end{array}$} \\
\hline & Esterified & Free & Total & \\
\hline $\mathrm{C}-$ & $1.11(0.02)$ & $0.48(-0.32)$ & $1.59(0 \cdot 19)$ & $1.44(0 \cdot 14)$ \\
\hline RS2- & $1.08(0.02)$ & $0.46(-0.34)$ & $1.54(0.18)$ & $1.61(0.20)$ \\
\hline RS3- & $1.07(0.02)$ & $0.48(-0.32)$ & $1.55(0.19)$ & $1.47(0.16)$ \\
\hline $\mathrm{C}+$ & $2.39(0.36)$ & $0.53(-0.29)$ & $2.91(0.45)$ & $0.76(-0.13)$ \\
\hline RS2+ & $2 \cdot 31(0.34)$ & $0.51(-0.30)$ & $2 \cdot 82(0 \cdot 43)$ & $0.79(-0.12)$ \\
\hline RS3+ & $2.27(0.33)$ & $0.48(-0.31)$ & $2.77(0.42)$ & $0.73(-0.15)$ \\
\hline SEM & $0.09(0.02)$ & $0.01(0.01)$ & $0.10(0.02)$ & $0.04(0.02)$ \\
\hline
\end{tabular}

Probability values for assessing specific comparisons between diet means $(P) \dagger$ :

\begin{tabular}{lcccc} 
Cholesterol & 0.0001 & NS & 0.0001 & 0.0001 \\
RS & NS & NS & NS & NS \\
$\begin{array}{c}\text { Cholesterol } \\
\times \text { RS } \ddagger\end{array}$ & 0.0001 & NS & NS & NS \\
\hline
\end{tabular}

C, control diet without added resistant starch (RS); RS2, diet with $6 \%$ RS type 2; RS3, diet with $6 \%$ RS type 3; -, diet without added cholesterol and cholic acid; +, diet with $1 \%$ cholesterol and $0 \cdot 1 \%$ cholic acid.

* Results are presented as means adjusted for initial body weight with standard errors of the means (SEM). Means of logarithms, adjusted for initial body weight, with their SEM are presented in parentheses. For details of diets and experimental procedures see Table 1 and pp. 193-194.

$\dagger$ Comparisons were performed in the logarithmic scale; no interaction implies similar proportional changes with RS at each level of cholesterol.

‡ Cholesterol, RS, main effect of cholesterol and enzyme-resistant starch addition on the measured variable; Cholesterol $\times \mathrm{RS}$, interaction between cholesterol and RS.

protocols of the Foundation for Scientific Research. Fortyeight male Wistar rats, weighing about $200 \mathrm{~g}$, were individually housed in wire-bottomed metabolism cages in a well ventilated room with the dark period from 20.00 to 08.00 hours. Cages were positioned on a rack and placed in front of tube lighting. Rats were allocated to the cages per group in a vertical manner in order to exclude mistakes in food administration and influences of temperature and lighting. Deionized water and food were given ad libitum. At the beginning of the experiment, animals were divided into two groups of twenty-four rats each, both groups having the same initial mean body weight. During the first 2 weeks one group received the normocholesterolaemic control diet (C-), containing neither added RS, nor cholesterol and cholic acid. The second group received the hypercholesterolaemic control diet $(\mathrm{C}+)$. At the end of this period both groups were divided into three subgroups of eight rats each, all having approximately the same initial mean body weight. One subgroup continued to receive the control diet $(\mathrm{C}-$ and $\mathrm{C}+$ ), while the other two groups were switched over to the test diets with $6 \%$ RS type 2 (RS2- and RS2+) or type 3 (RS3- and RS3+). All diets were fed for another 5 weeks. During this period water and food intakes were recorded daily. Faeces were collected daily during the last 3 weeks of the experiment. Faecal collections from each rat were pooled, freeze-dried, ground and analysed for steroid content. At the end of the experiment, rats were anaesthetized with diethyl ether and killed by decapitation. The liver was removed, blotted and stored at $-80^{\circ}$ until analysis for lipid content. Blood was collected in heparinized tubes, plasma prepared by centrifugation and stored at $-80^{\circ}$ until analysis for cholesterol content.

\section{Analytical methods}

The total dietary fibre content of the diets was determined according to the procedure of Prosky \& Lee (1992).

Plasma and liver lipids were extracted with chloroformmethanol $(2: 1, \mathrm{v} / \mathrm{v})$ and determined by TLC in combination with flame ionization detection (Ackman et al. 1990; De Schrijver \& Vermeulen, 1991). Free and esterified cholesterol, triacylglycerols and phospholipids were quantified after separation on chromarods (type-SIII) using petroleum ether-diethyl ether-formic acid (85:15:0.1, by vol.) as the solvent system. Plasma HDL- and LDL-cholesterol concentrations were measured enzymically with a Boehringer kit (Boehringer Pharma, Mannheim, Germany).

Individual faecal neutral steroids and bile acids were measured according to methods described by Miettinen et al. (1965) and by Grundy et al. (1965) respectively.

Total $3 \alpha$-hydroxy-bile acids were determined after extraction of the samples with $t$-butanol-water $(1: 1, \mathrm{v} / \mathrm{v})$. The extracted bile acids were quantified enzymically as described by Turley \& Dietschy (1978).

\section{Statistical analysis of data}

Experimental data are presented as means adjusted for initial body weight with SEM. Data were subjected to a logarithmic conversion in order to transform the observations to a scale of constant variance. Therefore, means of logarithms adjusted for body weight with standard errors of the logarithmic means are also presented. As both normoand hypercholesterolaemic diets were fed, data were subjected to a two-way ANOVA using the procedure of SAS (Statistical Analysis Systems, 1988) with cholesterol and RS consumption as main effects. The cholesterol $\times$ RS interaction was also tested. Initial body weight was also 
Table 3. Liver cholesterol concentrations ${ }^{\star}$ (Mean values with their standard errors for eight animals)

\begin{tabular}{|c|c|c|c|}
\hline \multirow[b]{2}{*}{ Diet } & \multicolumn{3}{|c|}{ Cholesterol concentration $(\mu \mathrm{mol} / \mathrm{g})$} \\
\hline & Esterified & Free & Total \\
\hline $\begin{array}{l}\text { C- } \\
\text { RS2- } \\
\text { RS3- } \\
\text { C+ } \\
\text { RS2+ } \\
\text { RS3+ } \\
\text { SEM }\end{array}$ & $\begin{array}{r}1.80(0.29) \\
1.78(0.29) \\
1.90(0.31) \\
75.30(1.85) \\
55.64(1.68) \\
58.61(1.64) \\
3.44(0.14)\end{array}$ & $\begin{array}{l}4.22(0.62) \\
4.29(0.63) \\
4.11(0.61) \\
6.56(0.81) \\
6.64(0.82) \\
6.47(0.81) \\
0.12(0.01)\end{array}$ & $\begin{array}{r}6.01(0.79) \\
6.10(0.79) \\
6.00(0.79) \\
81.85(1.89) \\
62.28(1.74) \\
65.07(1.72) \\
3.51(0.03)\end{array}$ \\
\hline \multicolumn{4}{|c|}{$\begin{array}{l}\text { Probability values for assessing specific comparisons between diet } \\
\text { means }(P) \dagger \text { : }\end{array}$} \\
\hline $\begin{array}{l}\text { Cholesterol } \neq \\
\text { RS } \ddagger \\
\text { Cholesterol } \\
\quad \times R S \ddagger\end{array}$ & $\begin{array}{l}0.0001 \\
\text { NS }\end{array}$ & $\begin{array}{l}0.0001 \\
\text { NS }\end{array}$ & $\begin{array}{l}0.0001 \\
\text { NS }\end{array}$ \\
\hline \multicolumn{4}{|c|}{$\begin{array}{l}\text { C, control diet without added resistant starch (RS); RS2, diet with } 6 \% \text { RS type } \\
2 \text {; RS3, diet with } 6 \% \text { RS type } 3 ;- \text {, diet without added cholesterol and cholic } \\
\text { acid; +, diet with } 1 \% \text { cholesterol and } 0 \cdot 1 \% \text { cholic acid. } \\
\text { * Results are presented as means adjusted for initial body weight with standard } \\
\text { errors of the means (SEM). Means of logarithms, adjusted for initial body } \\
\text { weight, with their SEM are presented in parentheses. For details of diets and } \\
\text { experimental procedures, see Table } 1 \text { and pp. } 193-194 \text {. } \\
\text { † Comparisons were performed in the logarithmic scale; no interaction implies } \\
\text { similar proportional changes with RS at each level of cholesterol. } \\
\text { †Cholesterol, RS, main effect of cholesterol and enzyme-resistant starch on } \\
\text { the measured variable; Cholesterol } \times \text { RS, interaction between cholesterol } \\
\text { and RS. }\end{array}$} \\
\hline
\end{tabular}

Table 4. Faecal excretion of neutral steroids* (Mean values with their standard errors for eight animals)

\begin{tabular}{|c|c|c|}
\hline \multirow[b]{2}{*}{ Diet } & \multicolumn{2}{|c|}{ Faecal excretion ( $\mu \mathrm{mol} /$ day) } \\
\hline & Coprostanol & Cholesterol \\
\hline $\begin{array}{l}\mathrm{C}- \\
\text { RS2- } \\
\text { RS3- } \\
\text { C+ } \\
\text { RS2+ } \\
\text { RS3+ } \\
\text { SEM }\end{array}$ & $\begin{array}{r}6.43(0.81) \\
6.68(0.83) \\
8.13(0.91) \\
31.53(1.49) \\
37.50(1.56) \\
44.11(1.60) \\
1.38(0.02)\end{array}$ & $\begin{array}{r}11.15(0.99) \\
10.72(0.97) \\
9.87(0.97) \\
278.64(2.44) \\
270.24(2.42) \\
254.51(2.40) \\
3.10(0.01)\end{array}$ \\
\hline \multicolumn{3}{|c|}{$\begin{array}{l}\text { Probability values for assessing specific comparisons between diet } \\
\text { means }(P) \dagger \text { : }\end{array}$} \\
\hline $\begin{array}{l}\text { Cholesterol } \neq \\
\text { RS } \ddagger \\
\text { Cholesterol } \times \text { RS } \ddagger\end{array}$ & $\begin{array}{c}0.0001 \\
\text { NS } \\
0.0001\end{array}$ & $\begin{array}{l}0.0001 \\
\text { NS } \\
\text { NS }\end{array}$ \\
\hline
\end{tabular}

C, control diet without added resistant starch (RS); RS2, diet with $6 \%$ RS type 2 ; RS3, diet with $6 \%$ RS type 3; -, diet without added cholesterol and cholic acid; +, diet with $1 \%$ cholesterol and $0.1 \%$ cholic acid.

* Results are presented as means adjusted for initial body weight with standard errors of the means (SEM). Means of logarithms, adjusted for initial body weight, with their SEM are presented in parentheses. For details of diets and experimental procedures, see Table 1 and pp. 193-194.

† Comparisons were performed in the logarithmic scale; no interaction implies similar proportional changes with RS at each level of cholesterol.

¥ Cholesterol, RS, main effect of cholesterol and enzyme-resistant starch on the measured variable; Cholesterol $\times \mathrm{RS}$, interaction between cholesterol and RS.

included in the model as a covariant to adjust to a common initial weight. Values were considered significantly different at the 0.05 level. Statistical comparison between results obtained in this study and the study reported previously (Vanhoof \& De Schrijver, 1997) was done after logarithmic conversion of the data. Data were subjected to three-way
ANOVA using SAS, with type of diet, cholesterol and RS as main effects. In addition, the interactions between type of diet and cholesterol, type of diet and RS and between cholesterol and RS were also tested.

\section{Results}

\section{Water intake, food intake and growth performance}

There were no significant differences for food and water intakes and growth rate with different diet regimens. Mean daily food and water intakes were $20.0 \mathrm{~g} / \mathrm{d}$ (SE 0.2) and $17.8 \mathrm{ml} / \mathrm{d}(\mathrm{SE} 0.4)$ respectively. The mean growth rate was $1.65 \mathrm{~g} / \mathrm{d}(\mathrm{SE} 0 \cdot 05)$.

\section{Plasma cholesterol concentrations}

There was no significant influence of RS consumption on free, esterified, and total cholesterol, nor was there any influence on HDL:LDL cholesterol ratios (Table 2). Addition of cholesterol and cholic acid to the test diets resulted in significantly higher concentrations of esterified, free and total cholesterol and in significantly lower HDL:LDL cholesterol ratios compared with the normocholesterolaemic animals. No significant interaction between RS and cholesterol supplementation on plasma cholesterol concentrations was found, indicating similar proportional changes due to RS consumption in normo- as well as hypercholesterolaemic rats.

\section{Liver cholesterol concentrations}

Table 3 shows the results of the effect of feeding RS on liver esterified, free and total cholesterol concentrations in normo- and hypercholesterolaemic rats. Feeding RS had no significant effect on the various cholesterol levels. However, consumption of RS types 2 and 3 resulted in a biologically important decrease in esterified and total cholesterol of 24 and $22 \%$ respectively. This was accompanied by a reduction in plasma esterified and total cholesterol of $4 \%$. Conversely, addition of cholesterol and cholic acid to the diets resulted in significantly higher liver cholesterol values. No significant interaction between RS and cholesterol supplementation on liver cholesterol concentrations was found.

\section{Faecal neutral steroid excretion}

Data on the influence of dietary supplementation of cholesterol and RS on faecal coprostanol and cholesterol excretion are presented in Table 4. No significant interaction between RS and cholesterol supplementation on faecal neutral steroid excretion was found. In addition, no statistically significant effect of RS consumption on cholesterol excretion was observed. However, RS consumption tended $(\mathrm{P}=0 \cdot 07)$ to cause an increase in faecal coprostanol excretion. Supplementation of the diet with RS type 3 caused a biologically important rise in coprostanol output of 25 and $40 \%$ in normo- and hypercholesterolaemic rats respectively. Faecal cholesterol output decreased by 4 and $9 \%$ under the same circumstances. RS type 2 increased the 
Table 5. Faecal bile acid excretion ( $\mu \mathrm{mol} / \mathrm{day})^{*}$

(Mean values with their standard errors for eight animals)



LCA, lithocholic acid; DCA, deoxycholic acid; CDCA, chenodeoxycholic acid; CA, cholic acid; $\alpha$-MCA, $\alpha$-muricholic acid; HDCA, hyodeoxycholic acid; UDCA, ursodeoxycholic acid; KLCA, 7- and 12-ketolithocholic acids; HCA, hyocholic acid; $\beta$-MCA, $\beta$-muricholic acid; $\omega$-MCA, $\omega$-muricholic acid; Total, sum of all individual bile acids mentioned; Total (enz.), $3 \alpha$-hydroxy-bile acids quantified as described on p. 194; Primary:secondary, (CA+ $\alpha$-MCA $+\beta$-MCA+HCA+CDCA): (LCA+DCA+HDCA+UDCA+KLCA+w-MCA); C, control diet without added resistant starch (RS); RS2, diet with 6 \% RS type 2; RS3, diet with 6 \% RS type 3; -, diet without added cholesterol and cholic acid; + , diet with $1 \%$ cholesterol and $0.1 \%$ cholic acid.

* Results are presented as means adjusted for initial body weight with standard errors of the means (SEM). Means of logarithms, adjusted for initial body weight, with their SEM are presented in parentheses. For details of diets and experimental procedures, see Table 1 and pp. $193-194$.

Table 6. The probability values from ANOVA for assessing specific comparisons of faecal bile acid excretions ${ }^{*} \dagger$

\begin{tabular}{lccc}
\hline Bile acid & Cholesterol & RS $\ddagger$ & Cholesterol $\times$ RS $\ddagger$ \\
\hline LCA & 0.0001 & 0.0122 & NS \\
DCA & 0.0001 & NS & NS \\
CDCA & 0.0001 & NS & NS \\
CA+ $\alpha$-MCA & 0.0001 & NS & NS \\
HDCA & NS & NS & NS \\
UDCA & 0.0001 & 0.0365 & NS \\
KLCA & 0.0001 & NS & NS \\
HCA & NS & NS & NS \\
$\beta$-MCA & 0.0001 & NS & NS \\
$\omega-$ MCA & 0.0001 & NS & NS \\
Total & 0.0001 & NS & NS \\
Total (enz.) & 0.0001 & 0.0693 & 0.0290 \\
Primary:secondary & NS & NS & NS
\end{tabular}

LCA, lithocholic acid; DCA, deoxycholic acid; CDCA, chenodeoxycholic acid; CA, cholic acid; $\alpha$-MCA, $\alpha$-muricholic acid; HDCA, hyodeoxycholic acid; UDCA, ursodeoxycholic acid; KLCA, 7- and 12-ketolithocholic acids; HCA, hyocholic acid; $\beta$-MCA, $\beta$-muricholic acid; $\omega$-MCA, $\omega$-muricholic acid; Total, sum of all individual bile acids mentioned; Total (enz.), $3 \alpha$-hydroxy-bile acids quantified as described on p. 194; Primary:secondary, $(\mathrm{CA}+\alpha-\mathrm{MCA}+$ $\beta-\mathrm{MCA}+\mathrm{HCA}+\mathrm{CDCA}):(\mathrm{LCA}+\mathrm{DCA}+\mathrm{HDCA}+\mathrm{UDCA}+\mathrm{KLCA}+\omega-\mathrm{MCA})$.

* For details of diets and experimental procedures, see Table 1 and pp. 193-194. † Comparisons were performed in the logarithmic scale; no interaction implies similar proportional changes with RS at each level of cholesterol.

$¥$ Cholesterol, RS, main effect of cholesterol and enzyme-resistant starch addition on the measured variable; Cholesterol, $\times$ RS, interaction between cholesterol and RS.

coprostanol and decreased the cholesterol excretion by $4 \%$ in normocholesterolaemic rats and with 19 and $3 \%$ in hypercholesterolaemic rats respectively. Hypercholesterolaemic rats had significantly higher faecal coprostanol and cholesterol excretion in comparison with rats receiving the normocholesterolaemic test diets.

\section{Faecal bile acid excretion}

Primary bile acid excretion was calculated as the sum of cholic acid, chenodeoxycholic acid, hyocholic acid and $\alpha$ and $\beta$-muricholic acids (Eyssen \& Robben, 1989). The secondary bile acids are $\omega$-muricholic acids, lithocholic acid, deoxycholic acid, hyodeoxycholic acid, ursodeoxycholic acid and 7- and 12-ketolithocholic acids.

Daily excretion of lithocholic acid and ursodeoxycholic acid was significantly higher when RS was fed (Tables 5 and 6$)$. This resulted in a tendency $(P=0 \cdot 07)$ towards a higher excretion of total bile acids. However, this increase was only found when total $3 \alpha$-hydroxy-bile acid excretion was determined enzymically.

Hypercholesterolaemic rats had higher faecal excretion of all individual bile acids, except hyocholic and hyodeoxycholic acids, as well as total bile acids compared with normocholesterolaemic groups. No effect of cholesterol and RS feeding on the primary:secondary bile acid ratio was observed.

\section{Discussion}

Addition of $6 \% \mathrm{RS}$ in combination with cholesterol and cholic acid to a conventional rat diet had no effect on daily food and water intakes or on daily growth. These observations agree with results obtained by Gee et al. (1991), de Deckere et al. (1993, 1995) and Schulz et al. (1993). Conversely, addition of $10 \%$ RS originating from highamylose corn starch or $20 \%$ resistant potato starch resulted in an increase in food intake of 14 and $36 \%$ respectively (Mallett et al. 1988). This might be due to the fact that the animals adapted to the lower energy content of the test diets. Also, higher growth values were observed in rats fed on a diet containing $10 \%$ retrograded corn starch (Faulks et al. 1989). Although semi-synthetic diets were used in all studies reported, the effect of RS on food intake and growth might depend on the source and dose of RS added to the diet. This might explain why rather different results were found.

Replacing highly digestible starch in the diet by RS can lower serum cholesterol concentrations in normo- as well as hypercholesterolaemic rats (de Deckere et al. 1993, 1995; Younes et al. 1995; Vanhoof \& De Schrijver, 1997). Dietary fibre in general, and consequently $\mathrm{RS}$ in particular, might influence the cholesterol metabolism through one or more of 
the following mechanisms: increased faecal bile acid excretion; altered intestinal absorption, metabolism and release of fat and cholesterol; reduced insulin-stimulated cholesterol synthesis; and the systemic effects of fermentation byproducts like propionic acid and their effects on hepatic cholesterol synthesis. It has been shown that RS has the capacity to form inclusion complexes with bile acids due to its helical structure (Abadie et al. 1994). However, not every type of RS can bind bile acids. It is not expected that RS in the shape of granules can bind bile acids. The fact that RS consumption influences bile acid solubility was indicated by the studies of van Munster et al. (1993, 1994) showing a decrease of soluble deoxycholic, lithocholic and chenodeoxycholic acids in the aqueous phase of stool of humans consuming a daily dose of $3 \times 15 \mathrm{~g}$ native uncooked high-amylose corn starch. In addition, an increase in primary bile acid excretion was observed in those volunteers consuming RS. Consequently, the cytotoxicity of the faecal aqueous phase decreased. Brown (1996) confirmed this. Adding $20 \%$ crude potato starch to a hypercholesterolaemic rat diet also resulted in lower plasma cholesterol concentrations $(-40 \%)$ due to enhanced cholesterol and bile acid excretion (Levrat, 1996). Besides, Verbeek et al. (1995) suggested that the cholesterollowering effect of retrograded high-amylose corn starch in rats may be explained by an increased influx of neutral steroids and bile acids into the caecum, an increased faecal excretion of bile acids, and/or an altered bile acid profile.

The observed reduction of plasma cholesterol through consumption of RS shown in the above-mentioned studies was not confirmed in the present experiment, neither for normo- nor for hypercholesterolaemic rats. This discrepancy may be due to the fact that in the present study conventional instead of semi-synthetic diets were used. Adding RS to a conventional diet resulted in lower free, esterified and total plasma cholesterol concentrations when compared with results obtained in our previous study in which rats were fed on the same sources of RS types 2 and 3 added to a semi-synthetic diet (Vanhoof \& De Schrijver, 1997). These semi-synthetic diets contained corn starch, beef tallow, casein, sucrose, and vitamins and minerals as the main ingredients $(60,14,17.8,3.8$ and $4.5 \%$ respectively). The RS types 2 and 3 sources replaced normal corn starch for 10.8 and $14.6 \%$ in the test diets containing RS, whereas $1 \%$ cholesterol and $0 \cdot 1 \%$ cholic acid replaced beef tallow in the hypercholesterolaemic diets.

Feeding semi-synthetic diets also resulted in liver total cholesterol concentrations approximately four times higher than the value in the present study for hypercholesterolaemic rats receiving the conventional diet. These observations can be caused by the lower digestibility of conventional diets compared with semi-synthetic diets. In addition, fermentation processes are less intensive when consuming conventional diets in comparison with semi-synthetic diets, since the effect of RS added to the conventional diet on caecal $\mathrm{pH}$ was less pronounced than for semi-synthetic diets. Also, adding RS to the conventional diet had no effect on caecal propionic and butyric acid concentrations whereas addition to a semi-synthetic diet significantly increased both values (results not published). Moreover, faecal cholesterol excretion was lower in rats receiving the normo- and hypercholesterolaemic semi-synthetic diets. Conversely, faecal excretion of coprostanol was higher under the same circumstances. Of the cholesterol excreted, 70 and $39 \%$ was converted into coprostanol in rats fed on the semi-synthetic diets, whereas feeding conventional diets resulted only in 43 and $12 \%$ conversion in normo- and hypercholesterolaemic rats respectively. Moreover, adding RS to hypercholesterolaemic semi-synthetic diets caused significantly higher coprostanol excretion and significantly lower total plasma cholesterol concentration. This significant effect was not confirmed in the present study. However, plasma cholesterol concentrations decreased by about $4 \%$ and coprostanol excretion increased by 19 and $40 \%$ when RS types 2 and 3 respectively were added to the hypercholesterolaemic conventional diet.

Higher daily excretion of bile acids was observed when conventional diets were fed rather than semi-synthetic diets. The effect on total $3 \alpha$-hydroxy-bile acid excretion was more pronounced in normo- than in hypercholesterolaemic animals. The faecal primary:secondary bile acid ratio was lower in rats fed on the semi-synthetic diets, indicating that proportionally less primary bile acids were converted to secondary bile acids due to less intensive fermentation processes in the large bowel when conventional diets were fed.

The tendency to higher faecal bile acid excretion and the lower liver cholesterol concentration in rats receiving the conventional diets supplemented with RS had no influence on plasma cholesterol concentration. This has also been observed in studies with other fibre sources (Ghoos et al. 1988; Illman et al. 1991).

Care must be taken in interpreting these results as not only the type and dose of the RS source added to the diet but also the type of dietary fat influences the effect of RS on cholesterol metabolism (de Deckere et al. 1993, 1995). Moreover, it is well known that the level of food intake can affect plasma cholesterol concentrations. Consequently, in some studies it is not clear whether RS as such is responsible for the cholesterol-lowering effect or not (de Deckere et al. 1993). Addition of $1 \%$ cholesterol and $0.1 \%$ cholic acid to the rat diet might in itself cause liver pathology (Dabai et al. 1996) and, consequently, it may be useful to evaluate this rat model to assess the hypocholesterolaemic effects of foods.

This study clearly indicates that the consumption of a conventional diet supplemented with RS has a different influence on cholesterol metabolism compared with semisynthetic diets. As a consequence, results obtained with semi-synthetic diets should be considered with caution as some metabolic effects might be affected by this particular dietary composition. Besides, the effect of RS added to the conventional diet might be masked due to the high levels of total dietary fibre in these diets (Table 1) which is much greater than the RS added. When considering the effect of RS consumption in humans, where dietary fibre intake is often very low, this overlapping of fibre and RS must be taken into account. Consequently, RS intake might influence cholesterol metabolism in humans taking a low-fibre diet.

Although the present data showed no significant effect of RS feeding on cholesterol metabolism in rats, these results may not reflect its effect in humans, particularly as rats have a different bile acid profile from humans (Heuman, 1989). 
As a consequence, further research is necessary to find out how cholesterol metabolism might be influenced in different species, including humans.

\section{References}

Abadie C, Hug M, Kübli C \& Gains N (1994) Effect of cyclodextrins and undigested starch on the loss of chenodeoxycholate in the faeces. Biochemical Journal 299, 725-730.

Ackman RG, McLeod CA \& Banerjee AK (1990) An overview of analyses by Chromarod-Iatroscan TLC-FID. Journal of Planar Chromatography 3, 450-462.

Beaulieu KE \& McBurney MI (1992) Changes in pig serum lipids, nutrient digestibility and sterol excretion during cecal infusion of propionate. Journal of Nutrition 122, 241-245.

Brown I (1996) Complex carbohydrates and resistant starch. Nutrition Reviews 54, S115-S119.

Chen W-J, Anderson J \& Jennings D (1984) Propionate may mediate the hypocholesterolaemic effects of certain soluble plant fibers in cholesterol-fed rats. Proceedings of the Society for Experimental Biology and Medicine 175, 215-218.

Dabai FD, Walker AF, Sambrook IE, Welch VA, Owen RW \& Abeyasekera S (1996) Comparative effects on blood lipids and faecal steroids of five legume species incorporated into a semipurified, hypercholesterolaemic rat diet. British Journal of Nutrition 75, 557-571.

de Deckere EAM, Kloots WJ \& van Amelsvoort JMM (1993) Resistant starch decreases serum total cholesterol and triacylglycerol concentrations in the rat. Journal of Nutrition 123, 2142-2151.

de Deckere EAM, Kloots WJ \& van Amelsvoort JMM (1995) Both raw and retrograded starch decrease serum triacylglycerol concentration and fat accretion in the rat. British Journal of Nutrition 73, 287-298.

De Schrijver R \& Vermeulen D (1991) Separation and quantification of phospholipids in animal tissues by iatroscan TLC/FID. Lipids 26, 74-76.

Eyssen H \& Robben J (1989) The indigenous microflora and the intestinal metabolism of cholesterol, bile acids and steroids. In The Regulatory and Protective Role of the Normal Microflora, pp. 71-88 [R Grubb, T Midtvedt and E Norin, editors]. Stockholm: Stockton Press.

Faulks RM, Southon S \& Livesey G (1989) Utilization of $\alpha$-amylase (EC 3.2.1.1)-resistant maize starch and pea (Pisum sativum) starch in the rat. British Journal of Nutrition 61, 291300.

Gee JM, Faulks RM \& Johnson IT (1991) Physiological effects of retrograded, $\alpha$-amylase-resistant cornstarch in rats. Journal of Nutrition 121, 44-49.

Ghoos Y, Rutgeerts P, Vantrappen G, Hiele M \& Schurmans P (1988) The effect of long-term fibre and starch intake by man on faecal bile acid excretion. European Journal of Clinical Investigation 18, 128-132.

Grundy SM, Ahrens EH \& Miettinen TA (1965) Quantitative isolation and gas-liquid chromatographic analysis of total fecal bile acids. Journal of Lipid Research 6, 397-410.
Heuman DM (1989) Quantitative estimation of the hydrophilichydrophobic balance of mixed bile salt solutions. Journal of Lipid Research 30, 719-730.

Illman RJ, Topping DL, Dowling K, Trimble RP, Russell GR \& Storer GB (1991) Effects of solvent extraction on the hypocholesterolaemic action of oat bran in the rat. British Journal of Nutrition 65, 435-443

Illman RJ, Topping DL, McIntosh GH, Trimble RP, Storer GB, Taylor MN \& Cheng B-Q (1988) Hypocholesterolaemic effects of dietary propionate: studies in whole animals and perfused rat liver. Annals of Nutrition and Metabolism 32, 97-107.

Levrat M-A, Moundras C, Younes H, Morand C, Demigné C \& Rémésy C (1996) Effectiveness of resistant starch, compared to guar gum, in depressing plasma cholesterol and enhancing fecal steroid excretion. Lipids 31, 1069-1075

Mallett AK, Bearne CA, Young PJ, Rowland IR \& Berry C (1988) Influence of starches of low digestibility on the rat caecal microflora. British Journal of Nutrition 60, 597-604.

Miettinen TA, Ahrens EH \& Grundy SM (1965) Quantitative isolation and gas-liquid chromatographic analysis of total dietary and fecal neutral steroids. Journal of Lipid Research $\mathbf{6}$, 411-424.

Prosky L \& Lee SC (1992) Enzymatic gravimetric AOAC/Prosky method for dietary fibre determination. In COST92, Metabolic and Physiological Aspects of Dietary Fibre in Food. Recent Progress in the Analysis of Dietary Fibre. Proceedings of a workshop held on 28 and 29 October 1994, Copenhagen, Denmark, pp. 113-118. Luxembourg: European Commission.

Schulz AGM, van Amelsvoort JMM \& Beynen AC (1993) Dietary native resistant starch but not retrograded resistant starch raises magnesium and calcium absorption in rats. Journal of Nutrition 123, 1724-1731.

Statistical Analysis Systems (1988) SAS User's Guide Statistics. Cary, NC: SAS Institute Inc.

Turley SD \& Dietschy JM (1978) Re-evaluation of the $3 \alpha$-hydroxysteroid dehydrogenase assay for total bile acids in bile. Journal of Lipid Research 19, 924-928.

Vanhoof K \& De Schrijver R (1997) Consumption of enzymeresistant starch and cholesterol metabolism in normo- and hypercholesterolemic rats. Nutrition Research 17, 1331-1340.

van Munster IP, Nagengast FM \& Tangerman A (1993) The effect of resistant starch on bile acid metabolism, cytotoxicity of faecal water and colonic mucosal proliferation. European Journal of Cancer Prevention 2, 12-13.

van Munster IP, Tangerman A \& Nagengast FM (1994) Effect of resistant starch on colonic fermentation, bile acid metabolism, and mucosal proliferation. Digestive Diseases and Sciences 39, 834-842.

Verbeek MJF, de Deckere EAM, Tijburg LBM, van Amelsvoort JMM \& Beynen AC (1995) Influence of dietary retrograded starch on the metabolism of neutral steroid and bile acids in rats. British Journal of Nutrition 74, 807-820.

Younes H, Levrat M-A, Demigné C \& Rémésy C (1995) Resistant starch is more effective than cholestyramine as a lipid-lowering agent in the rat. Lipids 30, 847-853. 